\title{
A Technique for Web Page Ranking by Applying Reinforcement Learning
}

\author{
Vivek Deshmukh \\ Student, \\ Department of Computer Engineering, \\ MIT Academy of Engineering, \\ Pune, India
}

\author{
S. S. Barve \\ Asst. Prof., \\ Department of Computer Engineering, \\ MIT Academy of Engineering, \\ Pune, India
}

\begin{abstract}
Ranking of site pages is for showing important web pages to client inquiry it is a one of the essential issue in any web search index tool. Today's need is to get significant data to client inquiry.

Importance of web pages is depending on interest of users. There are two ranking algorithm is utilized to demonstrate the current raking framework. One is page rank and another is BM25 calculation. Reinforcement learning strategy learns from every connection with dynamic environment. In this paper Reinforcement learning (RL) ranking algorithm is proposed. In this learner is specialist who learns through interactopm with dynamic environment and gets reward of an activity performed. Every site page is considered as a state and fundamental point is to discover score of website page. Score of website pages is identified with number of out connections from current website page .Rank scores in RL rank as considered in recursive way. Along these lines we can enhance outcomes with help of RL method in ranking algorithm.
\end{abstract}

\section{Keywords}

Ranking, Search engine, Agent, Value function, Reinforcement Learning, Artificial intelligence

\section{INTRODUCTION}

These days World Wide Web (WWW) is considered to be the best resource of data. It is because of simple access, minimal effort and being easily responsive to the user query [1]. Search engine is tools for discovering and getting access to the content available on the web. At every time clients look for data, enter their question in web search tool. The web search tools looks through pages and return a list of important ones. Web search tools include three preparing stages. The in the first place stage is called crawling. A crawler visits a site page, and takes after all the connections gave in that page. This operation creates a web chart (a web diagram comprises of hubs and edges, where hubs place for site pages and edges show the relations which are easy to get to from each one page to dissimilar pages). In the assembly web pages, content of each one page are analyzed to decide how it should be index (e.g. words are extracting from the titles, headings, or exceptional fields). Indexing allows data to be found as fast as possible. Ranking is the last stage. In this stage a large number of web pages were recorded in the past stage are filtered to discover matching cases for an requested inquiry and sorting them based on best related pages to users. Because of the huge volume of the web, very large number of applicable results is returned for a given inquiry. But users don't have the time and try to understand every one of the result. They regularly consider the main 10 or 20 results [2]. Because of that, ranking algorithm is needed. This algorithm improved searching to present the best related pages to users on their requested query.

Page Rank is one of the most significant ranking methods used in todays search engines. Not only is Page Rank a simple, robust and reliable way to measure the importance of web pages, but it is also computationally beneficial with admiration to other ranking methods in that it is query independent, and content independent. Otherwise said, it can be calculated offline using only the web graph1 structure and then used later, as users submit queries to the search engine, characteristically collective with other, query dependent rankings. In order to gauge the relative significance of web pages, they suggest Page Rank, a method for computing a ranking for each web page based on the graph of the web. Page Rank has application in search, browsing, and traffic estimation.

\section{A. Motivation}

The World Wide Web creates many new challenges for information recovery. It is extremely big and varied. Present approximations are that there are over 150 million web pages with a repetition life of less than one year. Additional significantly, the web pages are very diverse, ranging from "What is Joe having for lunch today?" to journals about information recovery. In adding to these main confronts, search engines on the Web must also contend with inexperienced users and pages engineered.

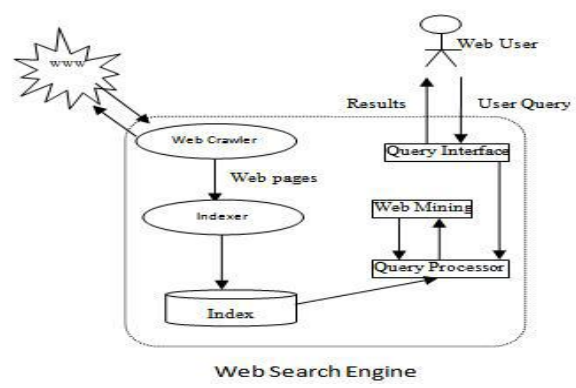

Fig. 1: Search Engine

Manipulate search engine ranking functions. However, unlike "at" document compilations, the World Wide Web is hypertext and gives considerable auxiliary information on top of the text of the web pages, such as link structure and link text. They take advantage of the link structure of the Web to produce a global importance" ranking of every web page. This ranking, called PageRank helps search engines and users rapidly make sense of the huge heterogeneity of the World Wide Web. 


\section{B. Background}

PageRank is one of the most significant ranking methods used in today's search engines. Not only is PageRank a simple, robust and reliable way to measure the importance of web pages, but it is also computationally beneficial with admiration to other ranking methods in that it is query independent, and content independent. Otherwise said, it can be calculated offline using only the web graph1 structure and then used later, as users submit queries to the search engine, characteristically collective with other, query-dependent rankings. In order to gauge the relative significance of web pages, they suggest PageRank, a method for computing a ranking for each web page based on the graph of the web. PageRank has application in search, browsing, and traffic estimation.

\section{LITERATURE SURVEY}

In this paper we survey on different web pages ranking algorithms.

In recent years, ranking based on learning has become a hot topic of research in the field of information retrieval [7]. Learning-based ranking methods are classified into three general categories: point-wise, pair-wise and list-wise methods. Point-wise approach is the simplest method to learn. The simple and common idea of this method is mapping each query-document pair to the numeric value assigned to the amount of their relevance. Linear regression (called REG) is a point-wise method based on statistics. REG aims to learn a linear ranking function in which the feature vector (multiple items) is mapped into the real value [8]. McRank method is based on the class of probabilities including incremental tree algorithm of multiple classification, ordered multiple classification and regression. This method uses combined ranking to minimize the number of pairs, which are ranked incorrectly [9]. A3CRank algorithm combines the results of algorithms like PageRank [10], BM25 [11] and TF-IDF [12], using the user`s feedback. This method is adaptive based on three components: connectivity, content and user behavior. The goal of agent is to maximize the number of clicks on the pages of high quality [13]. DistanceRank algorithm is based on learning used to receive penalty the logarithmic distances of pages. Its purpose is to minimize the total penalty. Distance calculation process continues until it converges to a constant value [14]. In $[15,16]$, Authors considered the problem of ranking in a graph-based data representation and proposed RL_Rank algorithm. This algorithm is based on the generalization of the reinforcement learning concepts for learning the ranking functions on graphs. RLRAUC ranking algorithm is based on reinforcement learning with point-wise approach and uses user feedback was proposed by Derhami et al [17]. The main idea behind the proposed method is based on consider value for each single word of query that is able to distinguish between relevant queries. FPR-DLA algorithm based on fuzzy logic and DLA is proposed to rank web pages. The algorithm consists of three stages. First, the weight of the links between web pages is determined using a set of learning automata. Secondly, the weight of each page is calculated. After weighting the web pages and links, web pages rank is calculated using a recursive formula. The higher weight of pages shows the greater user's interest in the pages [18]. Forsati and Meybodi presented a combination algorithm based on distributed learning automata and PageRank algorithm. The proposed algorithm uses simultaneously navigate information of user and link between pages in order to offer the pages to the user [19]. Yarahmadi proposed an algorithm in which a learning automata is assigned to each page the task of which is to learn the connection of each page with other pages. The proposed algorithm recursively obtains the rank of a learning automata derived from the ranks of the other automata [20]. Saati has proposed a method based on distributed learning automata. In this method, learning automata is assigned to each document the task of which is learning the connection of a document with other documents. Any document is displayed with a content vector of length equal to the number of subjects in the system [21]. In the pair-wise approach, document pairs are considered as sample of learning and problem of learning is formulated for ranking as a classifier. This approach extracts the document pairs from rank lists, and each document pair has a label which takes into account the partial correlation between the two documents. Then, the model trains classification with the labeled data and makes a classification model in ranking. Yule Freud et al. have proposed the RankBoost algorithm which is ranking based on learning that combines several ranking methods. This algorithm acts similar to AdaBoost algorithm for clustering document pairs. The only difference is their approach. RankBoost is defined on a pair of documents [22]

\section{PROPOSED WORK}

A. System Architecture

In Reinforcement learning main task is to balance exploration and exploitation. Exploration is finding out actions that was not been selected before and exploitation is sharing experience of tried action will be effective in reward. To select a best action from different possible action explore environment and exploit different experience to avoid punishment. Hence of balancing both is an important thing in RL.

Reinforcement learning is problem of learning through involvement with environment to achieve goal. In reinforcement learning, user means learner or decision maker is called as agent. The things interact with agent, is called environment. Agent selecting an action and reach to new situations to agent. So environment presents a reward, i.e. numerical value that has to maximize over time. There are elements of reinforcement learning system: a policy, a reward function and value function. 


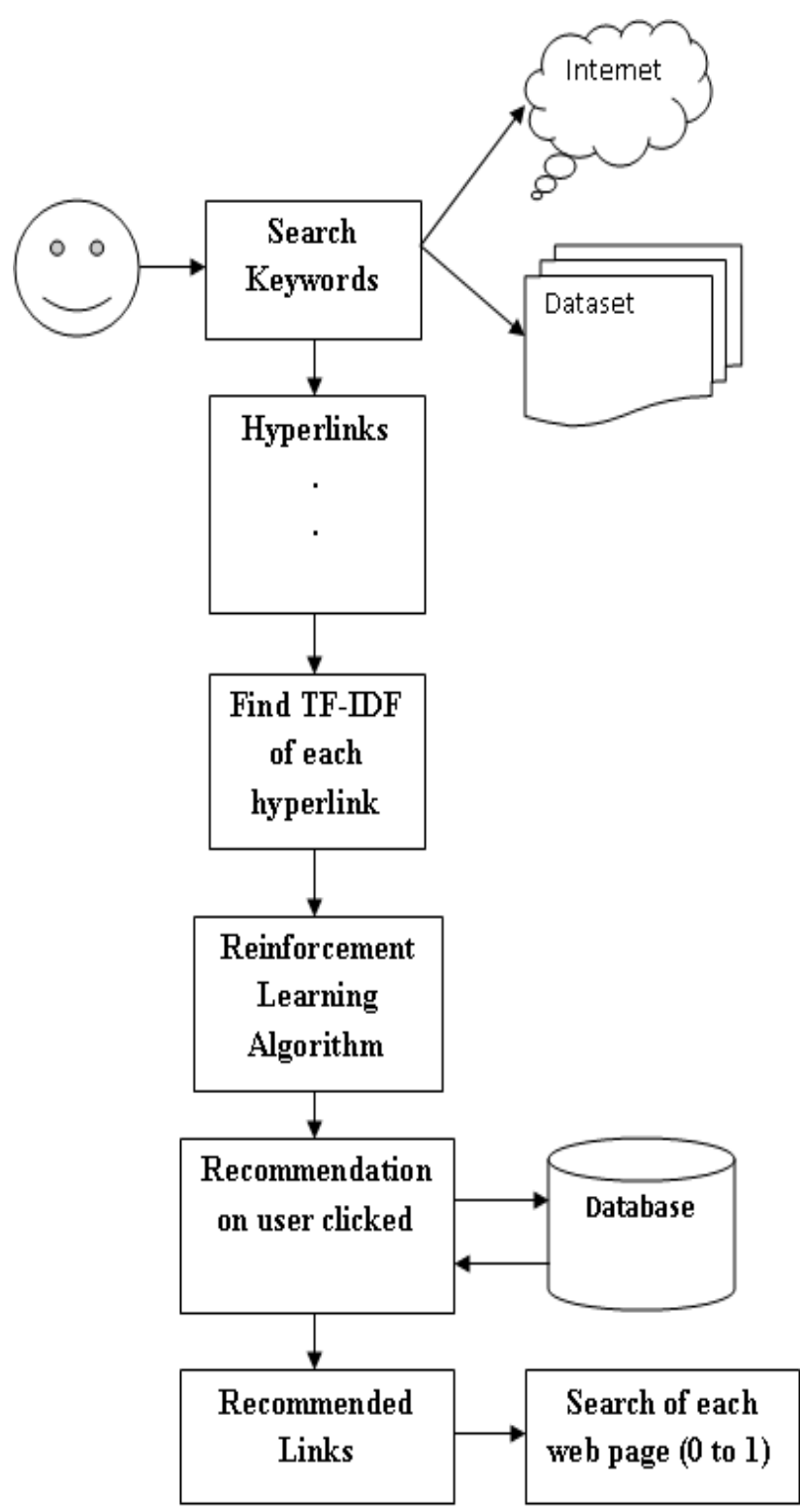

Fig 2: System Architecture

In the case of ranking of web pages agent is surfer who's traverse from one page to another page. Surfer interacts with environment i.e. with web pages and receives a reward. Probability of selecting a particular link on web page is called as policy of that state. Reward function is accumulating reward on during travelling through in short run while value function is the summing up of reward of each web page in long run.

\section{B. Proposed Algorithm}

The proposed algorithm is as follows:

1. Initialization:

2. Assume rank of all pages to be 1 .

3. Calculate rank of all pages by formula

4. $\quad \mathrm{PR}(\mathrm{T})=(1-\mathrm{d})+\mathrm{dPR}(\mathrm{T} 1) / \mathrm{O}(\mathrm{T} 1)+\ldots \mathrm{PR}(\mathrm{Tn}) / \mathrm{O}(\mathrm{Tn})(\mathrm{iii})$

5. Where,

6. $\mathrm{PR}(\mathrm{T})$ is the Rank of page A,

7. $\mathrm{PR}$ (TI) is the Page Rank of pages Ti which link to page A,
8. $\mathrm{O}(\mathrm{Ti})$ is the number of outbound links on page Ti and

9. $\mathrm{d}$ is a damping factor which can be set between 0 and 1

10. For every page $i \in \mathrm{V}$ (all web pages)

11. Probability of Selection of new links from page i.

12. Probnew (i) $=(\mathrm{d} \times \Sigma \operatorname{prob}(\mathrm{j}) / \mathrm{O}(\mathrm{j}))+(1 \mathrm{~d} / \mathrm{n})-(\mathrm{iv})$

13. $\mathrm{j} \in \mathrm{B}(\mathrm{i})$

14. For every page $\mathrm{p} \in \mathrm{V}$

15. Transition from page $i$ to page $j$ then score of that page is-

16. $\mathrm{r} \mathrm{ji}=1 / \mathrm{O}()$.

17. For calculation of new rank of page $i$

18. Rnew $(\mathrm{i})=\Sigma\{\operatorname{prob}(\mathrm{j}) / \mathrm{O}(\mathrm{j}) \times(\mathrm{rji}+\mathrm{Rt}(\mathrm{j})\}-(\mathrm{v})$

19. $\mathrm{j} \in \mathrm{B}(\mathrm{i})$

20. Update new rank for each click

21. $\mathrm{R} \leftarrow$ Rnew

C. Mathematical Model

TF_IDF:

TF_IDF, short for term frequency-inverse document frequency, is a numerical statistic that is intended to reflect how important a word is to a document in a collection or corpus. It is often used as a weighting factor in information retrieval and text mining. The tf-idf value increases proportionally to the number of times a word appears in the document, but is offset by the frequency of the word in the corpus, which helps to adjust for the fact that some words appear more frequently in general.

- Term Frequency:

In the case of the term frequency $\mathrm{tf}(\mathrm{t}, \mathrm{d})$, the simplest choice is to use the raw frequency of a term in a document

$$
t f t, d=0.5+0.5 \quad \frac{f_{t, d}}{\max \left\{f_{t^{\prime}, d}: t^{\prime} \in d\right\}}
$$

\section{- Inverse document frequency:}

The inverse document frequency is a measure of how much information the word provides, that is, whether the term is common or rare across all documents. It is the logarithmically scaled fraction of the documents that contain the word, obtained by dividing the total number of documents by the number of documents containing the term, and then taking the logarithm of that quotient.

$$
\text { idf } t, D=\log \frac{N}{|\{d \in D: t \in d\}|}
$$

\section{FUTURE DIRECTION}

Future direction is that used of both types of ranking algorithm in a one algorithm i.e. content based BM25 algorithm and connectivity based algorithm. Content based has low precision. It provides contents available on web are inconsistent and also give misinformation. Connectivity based is totally based on links between web pages. It counts votes of other pages to rank current web page. Main problem in connectivity based algorithm is web spamming. Due to 
web spamming other web pages are gain the benefit web traffic. So for that we used one solution to this problem that is combination of content and connectivity based algorithm which is hybrid algorithm which improved the quality of ranking results.

\section{CONCLUSION}

Current ranking algorithms, whether content-based or connectivity-based, suffer from low precision. Also they are not suitable for some situations and dependent on the context, they will work differently. In this paper, using the reinforcement learning concepts, we first proposed RL Rank algorithm which is a novel connectivity-based algorithm for ranking web pages. That's why utilizing the reinforcement learning ideas, we first proposed RL Rank algorithm which is a novel integration based algorithm for ranking pages.

\section{REFERENCES}

[1] Henzinger, M. R., Motwani, R., \& Silverstein, C. (2002). Challenges in web search engines. In ACM SIGIR Forum, vol. 36, no. 2, pp. 11-22.

[2] Barabási, A. L., \& Albert, R. (1999). Emergence of scaling in random networks, science, vol. 286, no. 5439, pp. 509-512.

[3] Agichtein, E., Brill, E., \& Dumais, S. (2006). Improving web search ranking by incorporating user behavior information. In Proceedings of the 29th annual international ACM SIGIR conference on Research and development in information retrieval (pp. 19-26). ACM, 2006.

[4] Joachims, T., Granka, L., Pan, B., Hembrooke, H., Radlinski, F., \& Gay, G. (2007). Evaluating the accuracy of implicit feedback from clicks and query reformulations in web search. ACM Transactions on Information Systems (TOIS), vol. 25, no. 2, pp. 7.

[5] Granka, L. A., Joachims, T., \& Gay, G. (2004, July). Eye-tracking analysis of user behavior in WWW search. In Proceedings of the 27th annual international ACM SIGIR conference on Research and development in information retrieval (pp. 478-479). ACM, 2004.

[6] Xue, G. R., Zeng, H. J., Chen, Z., Yu, Y., Ma, W. Y., Xi, W., \& Fan, W. (2004, November). Optimizing web search using web click-through data. In Proceedings of the thirteenth ACM international conference on Information and knowledge management (pp. 118-126). ACM, 2004.

[7] Qin, T., Liu, T. Y., Xu, J., \& Li, H. (2010). LETOR: A benchmark collection for research on learning to rank for information retrieval. Information Retrieval, vol. 13, no. 4 , pp. 346-374.

[8] Li, L., \& Lin, H. T. (2006). Ordinal regression by extended binary classification. In Advances in neural information processing systems (pp. 865-872), 2006.

[9] Li, P., Wu, Q., \& Burges, C. J. (2007). Mcrank: Learning to rank using multiple classification and gradient boosting. In Advances in neural information processing systems (pp. 897-904), 2007.

[10] Page, L., Brin, S., Motwani, R., \& Winograd, T. (1999). The PageRank citation ranking: Bringing order to the web, Technical Report. Stanford InfoLab, (pp.1-17, 1999, Available: http://ilpubs.stanford.edu:8090/422/.
[11] Robertson, S. E., \& Walker, S. (1994). Some simple effective approximations to the 2-poisson model for probabilistic weighted retrieval. In Proceedings of the 17th annual international ACM SIGIR conference on Research and development in information retrieval (pp. 232-241). Springer-Verlag New York, 1994.

[12] Salton, G., \& Buckley, C. (1988). Term-weighting approaches in automatic text retrieval. Information processing \& management, vol. 24, no. 5, pp. 513-523.

[13] Zareh Bidoki, A. M., Ghodsnia, P., Yazdani, N., \& Oroumchian, F. (2010). A3CRank: An adaptive ranking method based on connectivity, content and clickthrough data. Information processing \& management, vol. 46, no. 2, pp. 159-169.

[14] Zareh Bidoki, A. M., \& Yazdani, N. (2008). DistanceRank: An intelligent ranking algorithm for web pages. Information Processing \& Management, vol. 44, no. 2 , pp. $877-892$.

[15] Derhami, V., Khodadadian, E., Ghasemzadeh, M., \& Bidoki, A. M. Z. (2013). Applying reinforcement learning for web pages ranking algorithms. Applied Soft Computing, vol. 13, no. 4, pp. 1686-1692.

[16] Khodadadian, E., Ghasemzadeh, M., Derhami, V., \& Mirsoleimani, S. A. (2012). A novel ranking algorithm based on Reinforcement Learning. In Artificial Intelligence and Signal Processing (AISP), 2012 16th CSI International Symposium on (pp. 546-551). IEEE 2012.

[17] Derhami, V., Paksima, J., \& Khajeh, H. (2014). RLRAUC: Reinforcement learning based ranking algorithm using user clicks. In Computer and Knowledge Engineering (ICCKE), 2014 4th International eConference on (pp. 29-34). IEEE, 2014.

[18] Anari, Z., Meybodi, M. R., \& Anari, B. (2009). Web page ranking based on fuzzy and learning automata. In Proceedings of the International Conference on Management of Emergent Digital EcoSystems (p. 24). ACM, 2009.

[19] Forsati, R., Meybodi, M. R., \& Mahdavi, M. (2007). Web page personalization based on distributed learning automata. Proc. IKT, Ferdowsi University of Mashad, Mashad, Iran, 2007.

[20] Yarahmadi, T., Torkestani, J. A., \& Fatemeh, Z. (2012). A new method based on distributed learning automata for page ranking in web. International Journal of Physical Sciences, vol. 7, no. 13, pp. 2066-2075.

[21] Saati, S., \& Meybodi, M. R. (2006, May). Document Ranking using Distributed Learning Automata. In Proceedings of 11th Annual CSI Computer Conference of Iran, Fundamental Science Research Center (IPM), Computer Science Research Lab, Tehran, Iran, 2006.

[22] Phophalia, A. (2011, December). A survey on learning to rank (letor) approaches in information retrieval. In Engineering (NUiCONE), 2011 Nirma University International Conference on (pp. 1-6). IEEE, 2011 